\title{
Synthesis and Characterisation of CdS Nanoparticles in Mesoporous Copolymer Template
}

\author{
A. F. G. Monte ${ }^{a, b}$, N. O. Dantas ${ }^{b}$, P. C. Morais ${ }^{a}$, and D. Rabelo ${ }^{c}$ \\ ${ }^{a}$ Universidade de Brasília, Instituto de Física, Núcleo de Física Aplicada, 70919-970, Brasília-DF, Brazil \\ ${ }^{b}$ Universidade Federal de Uberlândia, Faculdade de Física, \\ Laboratório de Novos Materiais Isolantes e Semicondutores - LNMIS, 38400-902, Uberlândia-MG, Brazil and \\ ${ }^{c}$ Universidade Federal de Goiás, Instituto de Química, 74001-970, Goiânia-Go, Brazil
}

Received on 4 April, 2005

\begin{abstract}
Micron-sized spheres of mesoporous styrene-divinylbenzene (Sty-DVB) copolymer were produced by suspension polymerisation in the presence of inert diluents. Using these mesoporous microspheres as a template, optically stable CdS nanoparticles have been synthesized. To characterize these CdS nanoparticles, Raman spectroscopy and micro-photoluminescence were used. We have observed enhanced emission and lasing action at certain wavelengths that correspond to the whispering gallery (WG) modes of the microspheres. High optical stability and low threshold value make this optical system promising in microlaser applications.
\end{abstract}

Keywords: CdS nanoparticles; Raman spectroscopy; Micro-photoluminescence

\section{INTRODUCTION}

In recent years, the design and synthesis of nanometer-scale particles have been the focus of intense fundamental and applied research, with special emphasis on their size-dependent properties [1-6]. Current research interests include the development of new, nanostructured materials (specifically, semiconductor nanocrystal-quantum-dot based) for a broad range of applications: optoelectronics, telecommunications, sensors, and artificial photosynthesis. Using colloidal chemical syntheses, such nanoparticles, or nanocrystal quantum dots (NQDs), can be prepared with sub-nanometer precision having sizes from 10 to $100 \AA$. NQDs can be viewed as "quantum boxes" with precisely controlled dimensions and boundary conditions [2-5]. A spherical three-dimensional optical microcavity can be made of a nonabsorbing microsphere with a higher refractive index than the surrounding medium, having a diameter comparable to or slightly larger than the light wavelength, i.e., a few microns [7]. Several types of cavities have since been utilized to demonstrate nanocrystal QD lasing, including polystyrene microspheres [4], and distributedfeedback resonators [5]. In such microcavities, there exist a number of discrete resonant optical modes, the so-called whispering gallery modes (WGM) [7]. II-VI semiconductor compound QDs such as CdS can be grown in different matrices by different manufacturing processes [6]. When semiconductor QDs are embedded in the spherical microcavity, the QD luminescence can couple with the WGMs, and a lower threshold of stimulated emission or lasing modes of QDs may be realized. Due to the discrete structure of optical transitions in NQDs, thermal depopulation of the lowest "emitting" states is inhibited. Thus, NQDs are predicted to provide superior performance in lasing applications in comparison with bulk and other low-dimensional semiconductors.

In our work, micron-sized spheres (average diameters 20$100 \mu \mathrm{m})$ of mesoporous styrene-divinylbenzene (Sty-DVB) copolymer were used as a template to embed CdS nanoparticles. The Sty-DVB copolymer template has been of great interest because of its well defined porous structures, and it works as starting material for the synthesis of ion-exchange resins [9]. This copolymer template has been synthesized primarily as micron-spheres, making it ideal for studying microcavities.

As far as we know, this is the first time Sty-DVB works as a host for semiconductor nanocomposites. Previously, StyDVB has been used for hosting magnetite $\left(\mathrm{Fe}_{3} \mathrm{O}_{4}\right)$ nanoparticles [8]. To characterize these materials we have used Raman and photoluminescence spectroscopy.

\section{SAMPLE AND EXPERIMENTAL DETAILS}

The Sty-DVB copolymer used in this study was synthesized by suspension polymerization in the presence of inert diluents $[8,9]$. The solution containing the monomers (styrene-Sty and divinylbenzene-DVB), the diluents (toluene and heptane), and initiator (2,2'-azobisisobutyronitrile) was added to the aqueous phase at room temperature. The aqueous phase contained gelatine, ethylcellulose, and $\mathrm{NaCl}$. The flowchart in Fig. 1 explains the main steps. The polymerization occurs at $70^{\circ} \mathrm{C}$ and $400 \mathrm{rpm}$ (stirring speed) during 24 hours. After formation, the copolymer beads were then separated, washed with water/ethanol to remove the diluents, and vacuum dried.

Apparent density $\left(0.44 \mathrm{~g} / \mathrm{cm}^{3}\right)$, surface area $\left(140 \mathrm{~m}^{2} / \mathrm{g}\right)$, average pore diameter $(13 \mathrm{~nm})$, toluene regain $\left(1.52 \mathrm{~cm}^{3} / \mathrm{g}\right)$, heptane regain $\left(1.24 \mathrm{~cm}^{3} / \mathrm{g}\right)$, percentage of volume swelling in toluene (100\%), and percentage of volume swelling in heptane $(58 \%)$ were parameters used to characterize the spherical, micrometer-sized polymeric template. Sulfonation of the Sty-DVB spheres were performed using concentrated sulfuric acid ( $2 \mathrm{~g}$ of polymer $/ 30 \mathrm{~mL}$ of sulfuric acid). The reaction was carried out in the presence of dichloroethane (40\% in volume with respect to sulfuric acid). The Sty-DVB spheres were first suspended in dichloroethane for a few minutes. Then, sulfuric acid was added slowly while the temperature was maintained at $70{ }^{\circ} \mathrm{C}$ for $4 \mathrm{~h}$. The Sty-DVB polymer particles (200 $\mu \mathrm{m})$ average diameter) were separated by filtration, washed thoroughly with deionized water, and dried at $60{ }^{\circ} \mathrm{C}$ for 24 


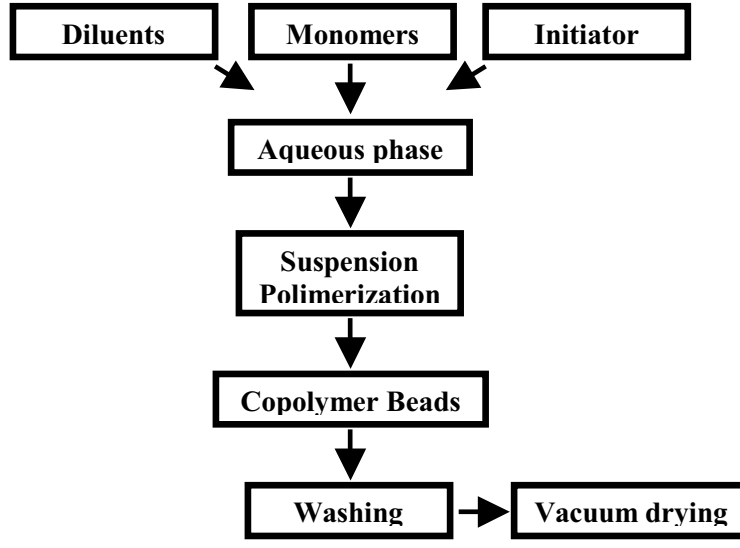

FIG. 1: Flowchart for preparation of copolymer micro-spheres.

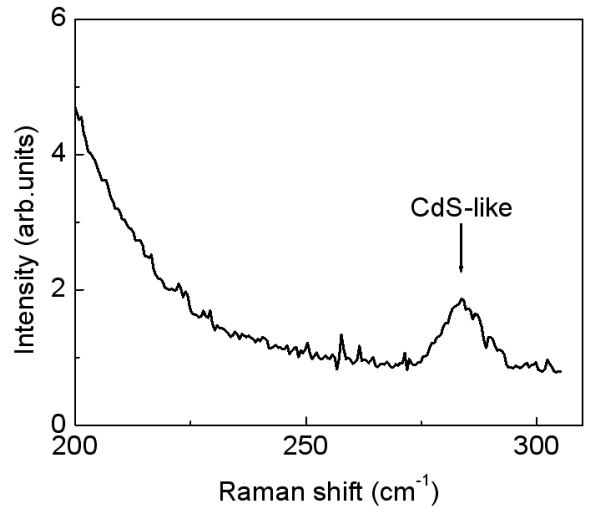

FIG. 2: Raman spectrum of CdS nanoparticles embedded in polymer microspheres measured at room temperature. The wavelength of excitation laser line is $488.0 \mathrm{~nm}$.

h. The ion-exchange capacity $\left(4.8 \mathrm{mmol}\right.$ of $\left.\mathrm{H}^{+} / \mathrm{g}\right)$ was determined as described in the literature [10]. CdS nanoparticles were embedded into the microspheres by ion-exchange. The microspheres used in the PL experiments showed a perfect surface with diameters varying from 20 to $100 \mu \mathrm{m}$.

To characterise the CdS nanoparticles, a microphotoluminescence $(\mu$-PL) setup has been used. The laser beam (excitation wavelength at $488 \mathrm{~nm}$; excitation power at $10 \mathrm{~mW}$ ) is focused onto a spot of about $3 \mu \mathrm{m}$ on the sample surface, but due to the spherical shape, the beam can be tightly focused inside the sample, providing a spot as low as $1 \mu \mathrm{m}$ wide. Raman scattering and photoluminescence (PL) spectra of the polymer microspheres embedded with CdS QDs are measured in a backscattering geometry by using a Jobin Yvon triple microraman system.

\section{EXPERIMENTAL RESULTS AND DISCUSSIONS}

The Raman spectra of the microspheres were measured as shown in Fig. 2 using the $488 \mathrm{~nm}$ excitation line of an argon ion laser. The Raman signals from the microsphere sample are strong for the CdS-like longitudinal optical (LO) phonon located at $280.3 \mathrm{~cm}^{-1}$, in agreement with what expected by the two-mode behavior of the lattice vibrations in $\mathrm{CdS}$ alloy.
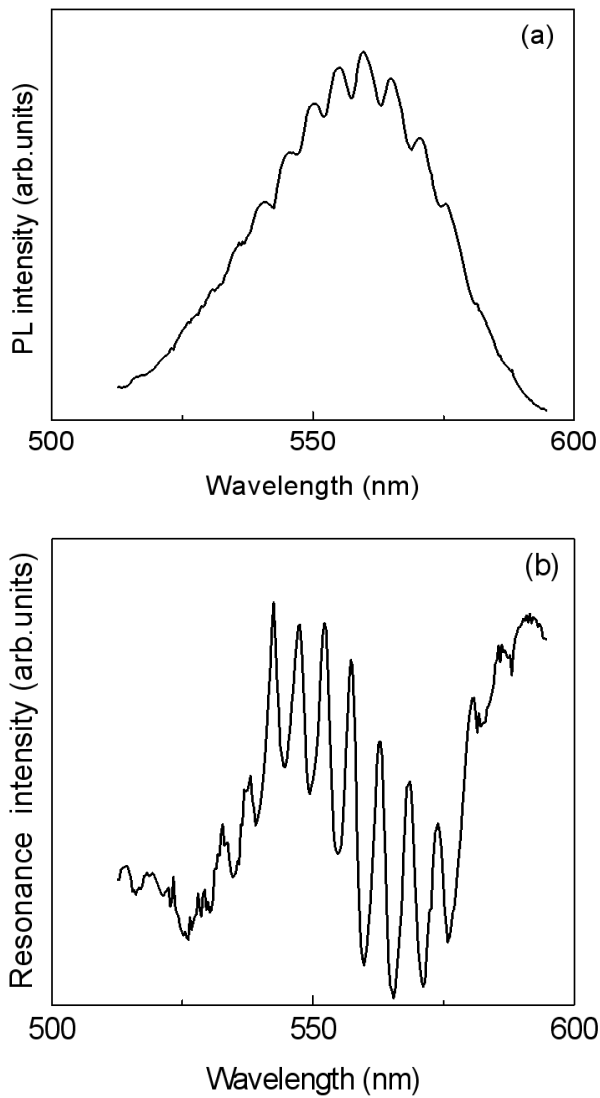

FIG. 3: (a) Low temperature (77 K) PL spectra of single polymer sphere. (b) Normalized photoluminescence spectrum shows whispering-gallery modes.

Figure 3a shows the emission spectrum of a single microsphere (average diameter of $40 \mu \mathrm{m}$ ). Details of the WG mode structure are shown separately in Fig. 3b after subtracting the Gaussian background PL spectrum. There is a clear periodical modulation which we assign to selected optical modes of the spherical microcavity.

One of the reasons to observe this phenomenon is due to the large interlevel spacing in NQDs, "quantum-confined" excitons are more robust than bulk excitons, allowing one to excite amplified spontaneous emission (ASE) at lower pump levels. Due to the discrete structure of optical transitions in NQDs, thermal depopulation of the lowest "emitting" states is inhibited. Therefore, NQDs are predicted to provide superior performance in lasing applications in comparison with bulk and other low-dimensional semiconductors. Also, the NQD gain medium was combined with an optical cavity that provided efficient positive feedback.

We have calculated the value of quality factor $Q$ using the following expression [11]: 


$$
\mathrm{Q}=\frac{\hbar \omega_{0}}{2 \hbar \gamma}
$$

where $2 \hbar \gamma$ is the Lorentzian fitting to the linewidth of the cavity modes and $\hbar \omega_{0}$ is the photon energy. At the wavelength of $552.1 \mathrm{~nm}(\hbar \omega=2.25 \mathrm{eV})$, the Lorentzian fit of the linewidth for the resonance mode is about $2 \hbar \gamma=0.0012 \mathrm{eV}$. Then, the quality factor is about $Q=1875$. From these results we can conclude that by embedding the CdS nanocrystals in the microsphere cavity, a strong coupling between photonic and electronic states have occurred.

\section{CONCLUSION}

In summary, optically stable CdS nanoparticles have been synthesized in mesoporous styrene-divinylbenzene copoly-

mer template. In addition, the $\mathrm{CdS}$ nanocrystalline system was used to quantitatively investigate the effects of lasing in microspheres. These efforts can be directed towards fabricating a tunable photonic crystal laser by incorporating QDs in photonic structures.

\section{Acknowledgement}

The authors acknowledge financial support from the Brazilian Agencies: Conselho Nacional de Desenvolvimento Científico e Tecnológico (CNPq), and Fundação de Amparo à Pesquisa do Estado de Minas Gerais (FAPEMIG).

[1] M. Kuwata-Gonokami and K. Takeda, Optical Materials 9, 12 (1998).

[2] Y. Yamamoto, R. E. Slusher, Phys. Today June, 66 (1993).

[3] S. I. Shopova, G. Farca, and A. T. Rosenbergera, W. M. S. Wickramanayake, and N. A. Kotov, Appl. Phys. Lett. 85, 6101 (2004).

[4] V. I. Klimov, and M. G. Bawendi, MRS Bulletin 26, 998 (2001).

[5] H.-J. Eisler, V. C. Sundar, M. G. Bawendi, M. Walsh, H. I. Smith, and V. I. Klimov, Appl. Phys. Lett. 80, 4614 (2002).

[6] N. F. Borrelli, D. W. Hall, H. J. Holland, and D. W. Smith, J. Appl. Phys. 61, 5399 (1987).
[7] Optical Processes in Microcavities, edited by R. K. Chang and A. J. Chamillo (World Scientific, Singapore, 1996).

[8] D. Rabelo, E. C. D. Lima, A. C. Reis, W. C. Nunes, M. A. Novak, V. K. Garg, A. C. Oliveira, and P. C. Morais, Nano Letters 1, 105 (2001).

[9] F. M. B Coutinho, D. Rabelo, Eur. Polym. J. 28, 1553 (1992).

[10] F. Helfferich, Ion Exchange, McGraw-Hill, New York, 1962.

[11] W. Barber and R. K. Chang, Optical Effects Associated with Small Particles (World Scientific, Singapore, 1998). 